\title{
Semi-Automated Development of Conceptual Models from Natural Language Text
}

\author{
Mussa Omar ${ }^{\mathrm{a}, 1}$ and George Baryannis ${ }^{\mathrm{b}}$ \\ a Department of Computing, University of Ajdabiya, Tripoli Road, Ajdabiya, Libya \\ ${ }^{b}$ Department of Computer Science, University of Huddersfield, Queensgate, Huddersfield, UK
}

\begin{abstract}
The process of converting natural language specifications into conceptual models requires detailed analysis of natural language text, and designers frequently make mistakes when undertaking this transformation manually. Although many approaches have been used to partly automate this process, one of the main limitations is the lack of a domain-independent ontology that can be used as a repository for entities and relationships, thus guiding the transformation process. In this paper, a semi-automated system for mapping natural language text into conceptual models is proposed. The system, called SACMES, combines a linguistic approach with an ontological approach and human intervention to achieve the task. SACMES learns from the natural language specifications that it processes and stores the information that is learnt in a conceptual model ontology and a user history knowledge database. It then uses the stored information to improve performance and reduce the need for human intervention. The evaluation conducted on SACMES demonstrates that: (1) by using the system, precision and recall for users identifying entities of conceptual models is increased by $6 \%$ and $13 \%$, respectively, while for relationships, increases are even higher, $14 \%$ for precision and $23 \%$ for recall; (2) the performance of the system is improved by processing more natural language requirements, and thus, the need for human intervention is decreased.
\end{abstract}

Keywords: Conceptual Modelling; Information Extraction; Natural Language Processing; Ontologies; Semi-structured data

\section{Introduction}

Conceptual Model development (CM) is the most important stage in the design of a system and database. There are as many as eighty different $\mathrm{CM}$ notations that can be used to describe requirement specifications [1]. To be considered qualified a CM must have the ability to reflect the real world environment [2]. Examples include the Entity Relationship Diagram (ERD) [3], and Unified Modelling Language (UML), which are the most commonly used in practice [4].

Despite their significance, however, designing CMs can be very problematic. Any errors in the design process will be costly to fix during implementation so correcting errors during the early stages of developing the model is considerably cheaper than correcting them at a later stage [5]. The process itself can face many difficulties, as identified by [6]:

1. Lack of domain knowledge, experience and formal training can cause difficulties in the creation of CMs, particularly for novice designers.

2. The complexity of relationships between the concepts of a CM can be very difficult for both novice and expert designers to identify in natural language text. This is because not every relationship mentioned in the requirement specification needs to be mapped into a relationship

\footnotetext{
${ }^{1}$ Corresponding author.

E-mail addresses: mussa.omar@uoa.edu.ly (M. Omar), g.bargiannis@hud.ac.uk (G.Baryannis)
} 
in the $\mathrm{CM}$ while, conversely, some relationships that are not mentioned in the requirements do need to be included.

3. There can be different solutions to the same problem, because CMs reflect the designer's viewpoint and how they interpret end user demands and goals. The fact that two points of view might be correct makes it very difficult to define one optimal solution for a problem.

In addition to the above problems, natural language, which is widely used for writing requirement specifications in industry, contains inherent problems, such as noise, silence, overspecification, contradiction, forward reference, wishful thinking and ambiguity. Also, as discussed in [7], natural language rules for conceptual model extraction are incomplete and overlapped, which means there is no reliable set of linguistic rules that can be used to transfer natural language specifications into a CM.

Because of the aforementioned difficulties faced by designers, technologies have become involved in conceptual model creation. However, there is no commercial or non-commercial tool which can automatically convert natural language text into a CM [6, 8]. Instead, various semi-automated approaches are used for this purpose, which includes, among others, linguistics-based, pattern-based, case-based and ontology-based approaches. While semi-automated, these approaches require considerable levels of human intervention and are not capable of learning from past examples and user behaviour to improve their performance.

This paper contributes to research efforts in extracting CMs from natural language text by developing a domain-independent semi-automated system to create CMs based on the ERD terminology and notation as introduced by Chen [3], in which human intervention can be further reduced by learning from the designers and from the natural language text that is processed. The system incorporates a linguistics approach, an ontological approach and Natural Language Processing (NLP). Human intervention is limited to three points in the process: (1) helping resolve ambiguities that prevent the system from deciding whether a noun is an entity, an attribute, or neither (e.g. in case of nouns unimportant to the domain or ones that model multi-valued attributes); (2) defining cardinalities and naming unnamed relationships; (3) reviewing the final results at the end of the process. Learning from past examples and user behaviour is achieved by storing entities and relationships obtained at the end of each mapping in a CM ontology and by storing designers' behaviour in a relational database. The model uses the information that is stored in the ontology and the database to improve its performance and to reduce the need for human intervention. To the best of the authors' knowledge, no system for extracting CMs from natural language text provides this capability.

Experiment results show that the system assists designers in creating improved CMs, with precision and recall for identifying entities increased by $6 \%$ and $13 \%$, respectively, with a $14 \%$ increase in precision for identifying relationships, and a $23 \%$ increase for recall, compared to not using the system at all and creating CMs manually. Also, the system is learning by processing more case studies: after being trained on fifty case studies in the experiments, it is capable of recalling $40 \%$ of entities in five additional (unseen) case studies, with a precision of $63 \%$ and $10 \%$ of relationships with a precision of $21 \%$, when it was unable to recognise any relationships and only $5 \%$ of entities without any training.

The proposed system can benefit software designers who use the ERD model by reducing the time and associated costs required to produce ERD-based CMs. This can potentially include industrial use cases and large-scale software engineering projects where providing a semi-automated solution would help speed up the process of creating models out of lengthy requirements documents. Additionally, the proposed system can be used as a learning tool for students in computing courses and other novice designers, by generating models out of a given natural language text and asking users to evaluate and adapt these models, facilitating their understanding of the ERD model.

The rest of this paper is organised as follows. Section 2 presents related work for extracting CMs from natural language text. Section 3 introduces the proposed system, while Section 4 demonstrates the system's evaluation. Conclusions and directions for future work are presented in Section 5.

\section{Related Work}


The most comprehensive literature reviews, to the best of our knowledge, classify the approaches that are used for extracting CMs from natural language into five main approaches, namely, the linguistics-based, pattern-based, case-based, ontology-based and hybrid (i.e. based on multiple techniques) approaches [6,9]. Table 1 illustrates a comparison between the different approaches used for extracting CMs from natural language text. In the sequel, we summarise some indicative works that follow these approaches.

Harmain and Gaizauskas [10] designed a natural-language-based case tool called Class Model Builder (CM-Builder) which is an example of the linguistics-based approach. It is intended to assist in extracting classes, attributes and relationships automatically from natural language specification text. In other words, it produces a class model representation, similar to that found in the Unified Modelling Language (UML). The CM-Builder works automatically but, like similar tools, it does require human intervention. There are two versions of CM-Builder: version 2 has a better performance profile and requires less human intervention than version 1 . The purpose of this work was not to produce a class model automatically from text, without human intervention, but to show that NLP can assist in producing an initial diagram, which can then be reconsidered and refined by the software engineer to produce a final version of a class diagram. CM-Builder is limited due to being based on Chen's rules [11] for analysing natural language specifications, since these rules cannot solve inherent ambiguity problems.

Table 1. Comparison between Approaches Used for Extracting Conceptual Models from Natural Language Specifications

\begin{tabular}{|l|l|l|l|}
\hline Approach & Examples & Advantages & Disadvantages \\
\hline $\begin{array}{l}\text { Linguistics- } \\
\text { based } \\
\text { approach }\end{array}$ & $\begin{array}{l}\text { CM-Builder [10], } \\
\text { ER-Converter [12] }\end{array}$ & Domain independent & $\begin{array}{l}\text { Does not include domain } \\
\text { knowledge and not } \\
\text { capable for solving } \\
\text { natural language } \\
\text { ambiguity }\end{array}$ \\
\hline $\begin{array}{l}\text { Pattern- } \\
\text { based } \\
\text { approach }\end{array}$ & $\begin{array}{l}\text { Modelling Wizard [13], } \\
\text { APSARA [14] }\end{array}$ & $\begin{array}{l}\text { Speeding up design via } \\
\text { reuse and improving } \\
\text { software quality by using } \\
\text { designs, which have } \\
\text { proved superior in } \\
\text { numerous applications. }\end{array}$ & $\begin{array}{l}\text { It is time consuming and } \\
\text { very difficult to build a } \\
\text { pattern library }\end{array}$ \\
\hline $\begin{array}{l}\text { Case-based } \\
\text { approach }\end{array}$ & $\begin{array}{l}\text { CSBR [15], } \\
\text { DES-DS [16] }\end{array}$ & $\begin{array}{l}\text { Benefits from reusing } \\
\text { previous designs. }\end{array}$ & $\begin{array}{l}\text { Developing conceptual } \\
\text { model libraries and } \\
\text { indexing mechanisms is } \\
\text { costly }\end{array}$ \\
\hline $\begin{array}{l}\text { Ontology- } \\
\text { based } \\
\text { approach }\end{array}$ & $\begin{array}{l}\text { OMDDE [17], } \\
\text { DC-Builder [18] }\end{array}$ & $\begin{array}{l}\text { Reusability of a knowledge } \\
\text { repository }\end{array}$ & $\begin{array}{l}\text { Development of both } \\
\text { domain-dependent } \\
\text { ontology and domain- } \\
\text { independent ontologies is } \\
\text { challenging }\end{array}$ \\
\hline $\begin{array}{l}\text { Hybrid } \\
\text { approach }\end{array}$ & EIPW and HBT [9] & $\begin{array}{l}\text { Using more than one } \\
\text { approach can help to avoid } \\
\text { some limitations of each } \\
\text { individual approach }\end{array}$ & $\begin{array}{l}\text { The approaches cannot be } \\
\text { integrated ideally }\end{array}$ \\
\hline
\end{tabular}

An indicative example of the patterns-based approach is the Modelling Wizard dialogue tool [13]. The tool stores numerous patterns, and an appropriate pattern is chosen in a stage-by-stage manner based on answers given to questions posed by users. The restriction of the tool is that extensive user intervention is needed for answering the questions, precluding and large-scale use.

The Management and Database Design Environment (OMDDE) [17] is an example of the ontologybased approach, an implementation of the authors' methodology for creating ontologies and validating entity relationship models [19]. The system was tested on beginner as well as on qualified designers. 
The results show that beginner designers who used the OMDDE system produced qualified CMs better than those who did not use the system. They also show that qualified designers who used the system produced a higher quality of CM than those who used a case tool such as the UML Case Tool and information sources such as Wikipedia. This work provides a good example of how ontologies can be used in extracting CMs. However, the authors used a lightweight, domain-dependent ontology for an auction, which means that the system is unlikely to work properly in different domains. Although the system allows more ontologies to be added and existing ontologies to be updated, it will require considerable effort and expertise in the knowledge base to achieve this.

Entity Instance Pattern WordNet (EIPW) follows the hybrid approach. Thonggoom, Song, and An developed an automated methodology for building Entity Instance Patterns (EIP) and Relationship Instance Patterns (RIP) repositories from previously designed databases [20]. These repositories are integrated with WordNet ontology (ontology approach), NLP techniques (linguistic approach) and human intervention to develop EIPW. The process is started by inserting natural language specifications into EIPW, which then uses part-of-speech tagging as a NLP technique for defining a list of noun phrases as candidate entities. EIPW then uses WordNet, human intervention and a knowledge base represented in EIP and RIP to extract entities and relationships as pre-requirements for the CM. Extracted entities and relationships are inserted into the EIP and the RIP repositories respectively to keep them updated. One of the limitations of EIPW is that it is not clear how the EIP and RIP repositories are structured and organised. It is also unclear to what extent the updated repositories will continue to capsulise and abstract properly.

Herchi and Abdessalem [18] proposed a tool called DC-Builder that also integrates the linguistics and ontology approaches in order to produce a class diagram from natural language specifications. The DC-Builder includes three stages. The first stage employs General Architecture for Text Engineering (GATE) as a NLP toolkit to discover sentence subjects, objects and verbs. In the second stage, rules for extracting class diagram elements from natural language are employed. The DC-Builder employs Chen's rules to define the main elements of the class diagram. The third stage is called refinement. The output from the second stage contains many elements that may not be entities, but are included because of applying Chen's rules. Using a domain entity can reduce the number of elements by keeping only nouns with potential for inclusion in the class diagram. The DC-Builder is evaluated using case studies from object-oriented analysis books and is shown to achieve a better performance than the CM-builder. The DC-Builder uses a domain-dependent ontology, though the authors do not mention which domain was used to provide domain knowledge for the DC-Builder. The reliance on a domain-dependent knowledge base may be considered a limitation of the DC-Builder.

Examples of more recent attempts to extract CMs from natural language follow. The advantages and disadvantages stated in Table 1 also apply to these. Al-Btoush [21] follows the linguistics-based approach, aiming to translate each part of speech in natural language specifications into a component of an ERD. Relying only on natural language techniques and linguistics rules is the main limitation of the tool, as these are not enough to tackle ambiguity in natural language and to eliminate improbable ERD components. A knowledge base and a minimum level of human intervention are necessary.

Combining linguistic and pattern-based approaches, Ben Abdessalem Karaa et al. [22] proposed Automatic Builder of Class Diagram (ABCD), a tool for mapping natural language text into a class diagram in three stages: pre-processing, syntactic feature extraction and extraction of class diagram elements. The Stanford Parser is used to achieve the first two stages, while patterns are used for the third. The authors defined more than 1000 patterns, which highlights the disadvantages of this approach: creating the patterns list is time-consuming and since the list cannot be exhaustive, it will require continuous updates every time a new domain is modelled.

The approach by Song et al. [6] follows similar steps to the aforementioned ones, but places greater emphasis on analysing and refining the natural language text to minimise conceptual errors that may result from incomplete or incorrect input. Also, an extensive comparative analysis that goes beyond precision and recall measurements is proposed to evaluate the generated conceptual models. However, there is no implemented prototype that applies this approach.

We believe that integrating approaches can help in solving the limitations which appear when each approach stands alone. For example, a linguistic approach is domain independent but it does not include a domain knowledge base. In addition, the approach faces difficulties in solving natural language ambiguities. Thus, it is a good idea to add a domain knowledge base, essentially combining linguistics 
and ontologies. Conversely, knowledge-based approaches such as the pattern-based, case-based and ontology-based ones depend on a set of linguistic rules extracted from a linguistic approach to guide the process of extraction of CMs from natural language text, since there is no domain-independent knowledge designed to support the creation of CMs. Furthermore, because of natural language ambiguities, and the fact that fully-automated extraction of $\mathrm{CMs}$ from natural language is not possible $[6,8]$, hybrid approaches need to be supported by a minimum level of human intervention to help in solving ambiguities in natural language text. A hybrid approach supported by a minimum level of human intervention would therefore help in producing a semi-automated tool to guide the process of extracting and producing CMs from natural language specifications. The proposed system, analysed in the following section, investigates this claim, focusing on providing capabilities, such as learning from past examples and user behaviour, that can minimise human intervention.

\section{Semi-automated Conceptual Model Extraction System (SACMES)}

SACMES relies on the integration of a linguistics-based approach with an ontology-based approach. These approaches are supported by a minimum level of human intervention to resolve natural language ambiguities. Moreover, because the ontology of a specific domain will not be sufficient to produce suitable reusable knowledge to support the creation of $\mathrm{CMs}$, a domain-independent ontology is needed. However, building a domain-independent ontology or an upper level ontology is challenging and time consuming, and requires domain knowledge expertise. Therefore, our intention is to fill this gap by building a domain-independent ontology which can be updated from the natural language specification text that is inserted into the proposed system. As the ontology is updated, it should be increasingly capable of providing useful knowledge to guide and support the process of conceptual model extraction from natural language text.

Figure 1 illustrates the architecture of the system. The system integrates NLP tools, WordNet ontology, linguistic rules, a Conceptual Model Ontology (CMO), and a User History Knowledge Base (UHKB) to help designers produce CMs from natural language text. A brief description of SACMES follows, while a more detailed analysis is offered in the remainder of this section. The input to the system is a requirement specification text that describes a specific problem. This is fed to a preprocessing stage, where NLP tools (1) are used to identify noun phrases that are included in the text. NLP along with the WordNet ontology help in eliminating nouns and noun phrases that are unlikely to be entities. Furthermore, NLP helps in identifying relationships between entities by using Stanford typed dependencies.

The second stage is entities identification, which is realised by using both CMO and the WordNet ontology (2) to distinguish between nouns that can be mapped into entities and those that are unlikely to be mapped into entities. Human intervention is required in this stage in order to apply the domainimportance $^{2}$ and multi-attributes linguistic rules $^{3}$. The third and final stage is relationships identification, which again depends on CMO and human intervention. In this case, users help to apply the need-to-know rule ${ }^{4}$. Any user interaction in both of these stages is recorded in UHKB.

The outputs of the system are a CM and user behaviour. After the CM has been viewed by the user, it is inserted into the CMO in order to update the ontology and increase its ability to provide relevant information and guide future users in the creation of CMs. The user behaviour is also inserted into the

2 If a noun phrase is important within a requirement specification, then it can be mapped into an entity, e.g. a doctor or a patient within a hospital-related specification.

3 If a noun phrase represents attributes with multiple values, then it can be mapped into an entity, e.g. telephone number.

4 The need-to-know rule specifies that if a verb represents a relationship between entities that need to be remembered in a problem specification, then there is a relationship between the entities. For example, the sentence 'Each plant is divided into departments' indicates that there is a relationship between 'plant' and 'departments', the relationship being that each plant is divided into departments. It is therefore important to know and remember how many departments each plant is divided into and to which plant each department belongs. 


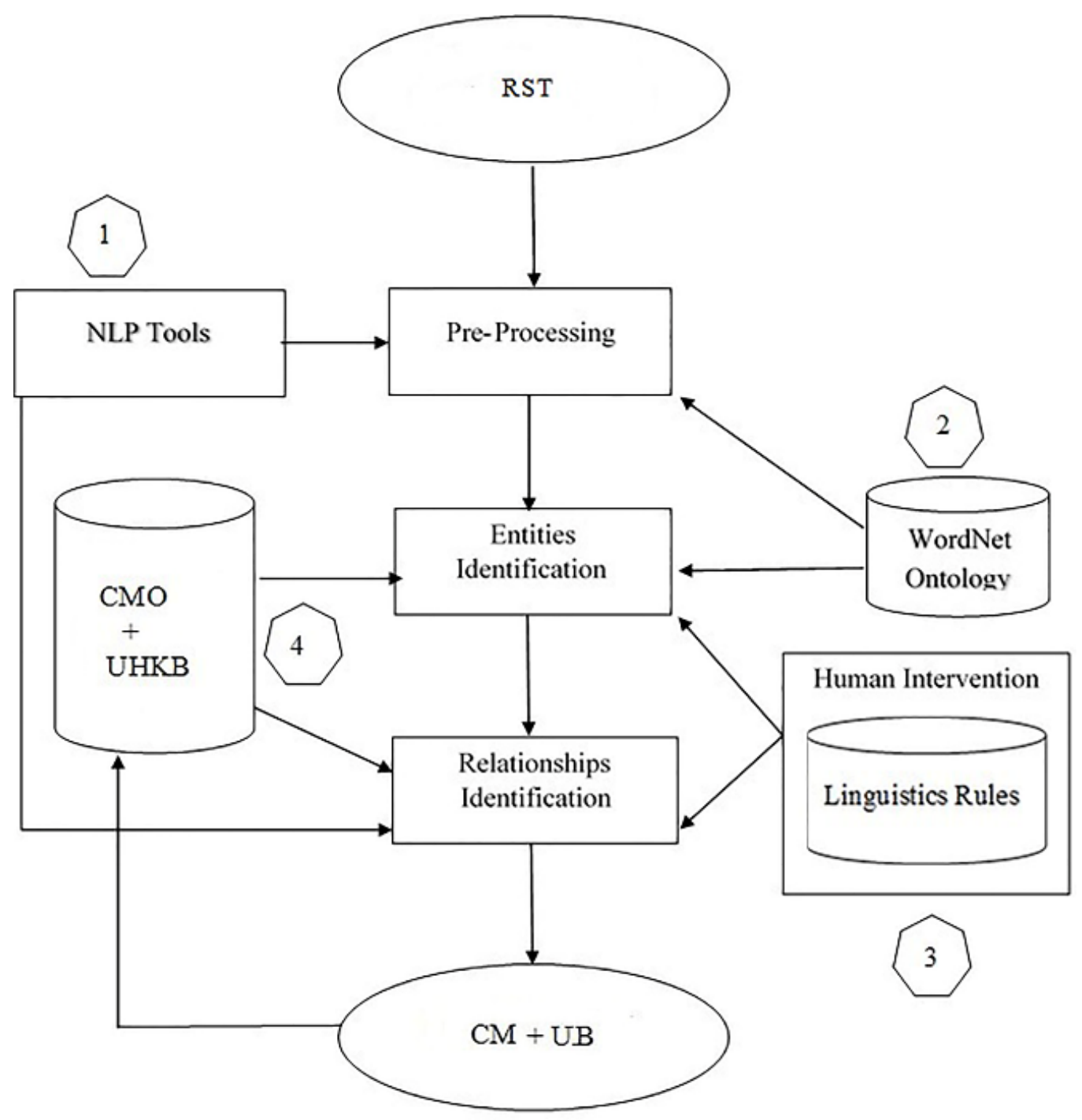

Fig. 1. SACMES Architecture

UHKB to update it. The system then uses this knowledge to inform the user when a similar situation to those stored in the UHKB is processed.

\subsection{Conceptual Model Ontology (CMO)}

The CMO learns from natural language requirements and uses this information to support users when a similar scenario is processed. Figure 2 shows the CMO hierarchy. Entities in the CMO are divided into three groups, namely, strong entities, mid-entities and other entities (entities that have been defined by designers but which do not belong in the strong or mid-entities groups). Each group is further divided into subgroups. The entities added by the system are introduced into these subgroups, whereas relationships are added under the object properties hierarchy. The CMO hierarchy is adapted from WordNet, which divides nouns into strong entities, mid-entities and weak entities. Weak entities are not considered as entities and therefore are not added into the CMO hierarchy. 


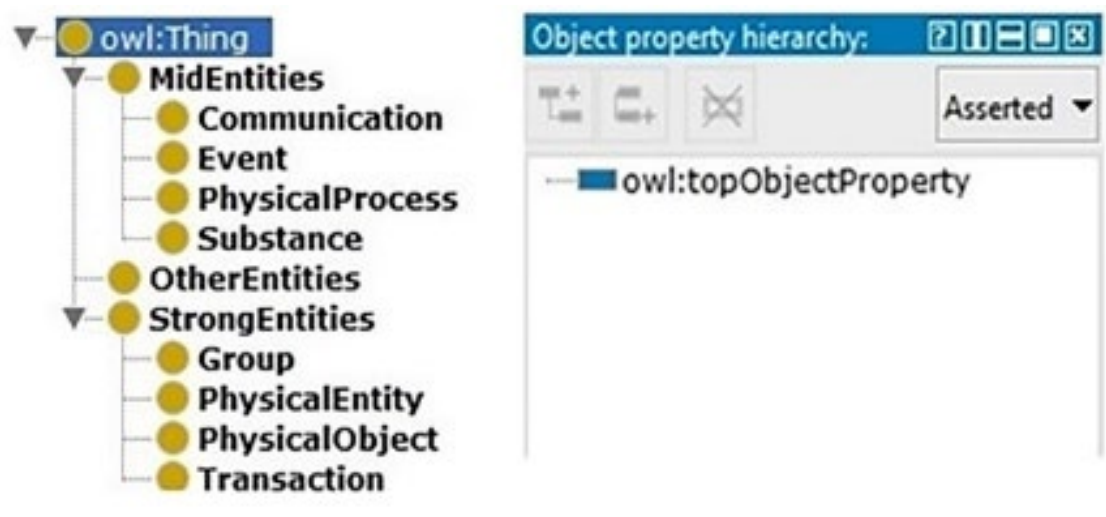

Fig. 2. CMO Hierarchy

\subsection{User History Knowledge Base (UHKB)}

The UHKB database records users' behaviour when using the system and utilises this history to guide subsequent users in extracting CMs. The UHKB is a relational database (built on Microsoft SQL Server) and its schema is shown in Table 2.

Table 2. UHKB Database Schema

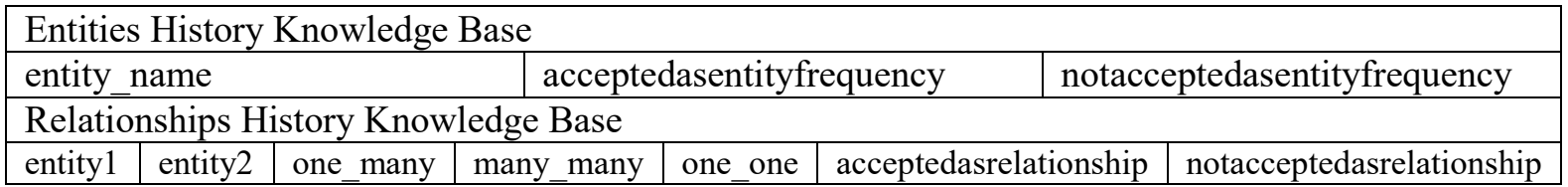

The Entities History Knowledge Base (EHKB) table records users' behaviour with regard to entities, while the Relationships History Knowledge Base (RHKB) stores users' behaviour regarding relationships. Both EHKB and RHKB are initially empty and are populated each time user intervention is requested. In the remainder of this section, we describe how EHKB is populated and used.

When the system encounters a noun and is unsure about whether it should be included as an entity in the CM, it looks into EHKB. If it is empty, user intervention is immediately requested, and the user's decision is recorded by adding an entry to EHKB for this particular noun, with either acceptedasentityfrequency or notacceptedasentityfrequency equal to 1, depending on the user's decision. The next time this particular noun is encountered, SACMES looks into EHKB, finds the relevant entry and informs the user that the majority of past occurrences resulted into accepting the noun as an entity (because acceptedasentityfrequency is greater than notacceptedasentityfrequency). The user is then free to decide: they can either accept the recommendation, or reject it. This decision is then recorded by updating the frequencies, increasing or decreasing values as necessary. In subsequent occurrences, the recommendation made by SACMES again depends on the majority. If the two frequencies are equal, then no recommendation is made and the user has to make the decision on their own.

RHKB works in a similar manner, though for each entry more information is stored: the names of the two entities participating in the relationship and frequencies for accepting or not accepting the relationship and, if accepted, frequencies for the associated cardinality. Each time user intervention is required, if the user decides to accept the relationship, they need to also define cardinality restrictions. The system proposes a particular cardinality restriction in the same way that it proposes to accept or reject the relationship: by returning the cardinality whose frequency is higher. Again, if the highest cardinality frequency is shared between two or more cardinalitt restrictions, SACMES does not provide any recommendation. Also, no cardinality restriction is recommended when the notacceptedasrelationship frequency is higher than the acceptedasrelationship frequency. 


\subsection{Pre-Processing Stage}

Flow Chart Keys:

RST: Requirement Specification

Text.

NPs: Noun Phrases.

CAN: Compound Attributes Nouns. CEs: Candidate Entities
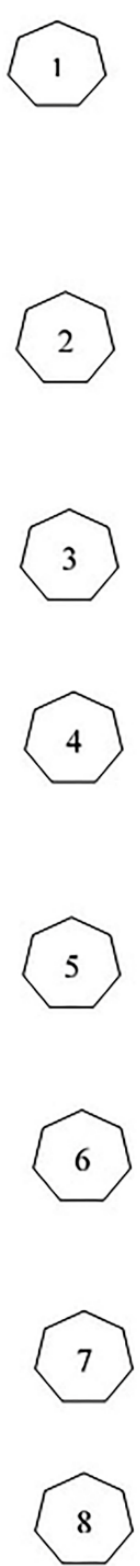
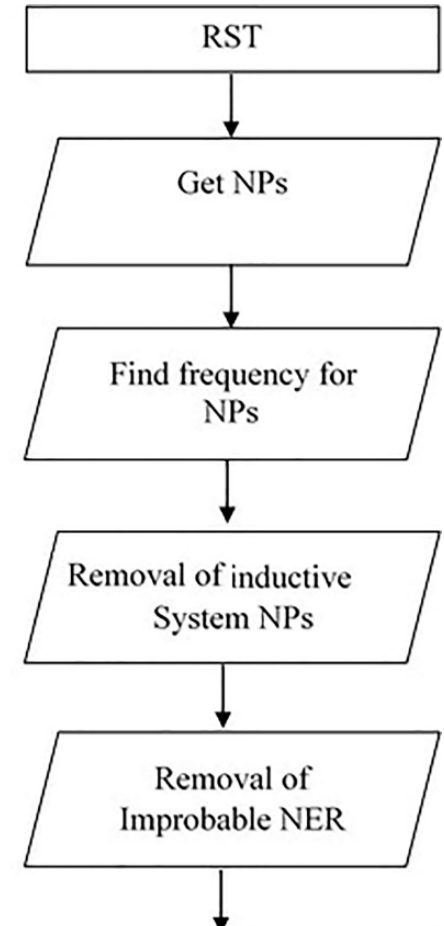

Removal of Inductive of attribute NPs
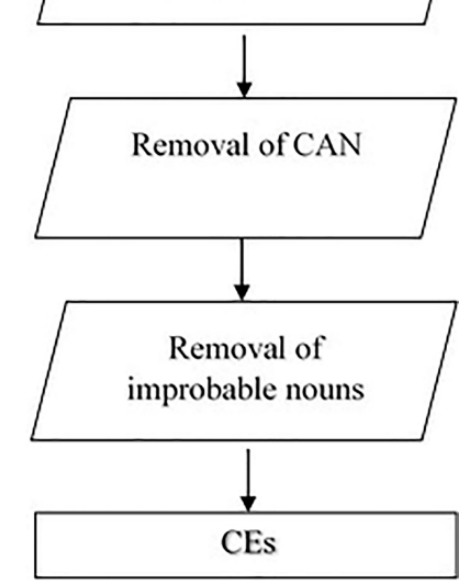

Fig. 3. Flow Chart of Pre-Processing Stage

Figure 3 illustrates the pre-processing stage. In this stage, Stanford CoreNLP ${ }^{5}$ [23] is employed to achieve natural language tasks for SACMES. This stage includes the following steps:

1. The system uses the Stanford PoS $\operatorname{tagger}^{6}$ [24] to define noun phrases (NPs) from the requirement specification text.

2. The system defines a frequency for each Noun Phrase (NP). The frequency refers to how many times a noun phrase is mentioned in the requirement specification text.

3. Removal of system indicative nouns. Some nouns, such as 'system', 'database', 'record' and 'application' are indicative of the system [25]. These nouns are removed from the NPs list. The system uses string matching to eliminate such nouns.

${ }^{5} \mathrm{https}: / /$ stanfordnlp.github.io/CoreNLP/

${ }^{6} \mathrm{https}: / /$ nlp.stanford.edu/software/tagger.shtml 
4. Removal of improbable NER classes. The system uses the Stanford Name Entities Recogniser ${ }^{7}$ [26] to exclude nouns that are indicative of being an organisation, location, person, percentage or time from being tabled. Logically, these classes would not normally be mapped into entities.

5. Removal of NPs indicative of attributes. The system uses a predefined list of nouns which are indicative of attributes. This list includes name, birthdate, number, gender, size, colour, age, username, password, date and year, month and day. If a NP matches any of this list, then it is removed.

6. Removal of Compound Attribute Nouns (CAN). For example, 'student number' is a noun phrase made of two nouns. The second noun is indicative of an attribute, and therefore such nouns are removed from the noun phrase list.

7. Removal of improbable nouns. In some cases, the PoS tagger defines improbable nouns. The system uses NPs found in WordNet 3.1 as standard. Each NP in the candidate list is matched against the WordNet list. If it is not matched, then it is removed. Sometimes a NP is made of compound nouns. In this case, the system divides a compound noun into separate nouns and matches each one separately against WordNet. All sub-nouns within the compound nouns must match; otherwise, the NP is removed from further consideration.

8. The NPs remaining after completion of these steps are considered Candidate Entities (CEs). The CEs are the output of the pre-processing stage and the input for the entities identification stage.

\subsection{Entities Identification Stage}

Figure 4 illustrates the entities identification stage which includes the following steps:

1. The process starts by searching for each item on the candidate entities list in the conceptual model ontology. If a candidate entity is found in the ontology, then it is considered as an entity.

2. WordNet is used to find a hypernym chain for each candidate entity that is not found in the ontology. If the hypernym chain of a candidate entity matches the strong entities group, then the candidate entity is considered an entity and inserted into the entities list.

3. If the hypernym chain of a candidate entity does not match the strong entities group but matches the mid-entities group, then the system uses the EHKB and linguistic rules either to discard the candidate noun or to accept it as an entity (using human intervention).

4. If the hypernym chain of a candidate entity does not match the strong or mid-entities groups, but does match the weak entities group, then the candidate entity is removed from the candidate entities list.

5. If the hypernym chain of a candidate noun does not match the strong entities, mid-entities or weak entities groups, and its frequency is equal to one, then the candidate entity is removed from the candidate entities list.

6. If the hypernym chain of a candidate noun does not match the strong entities, mid-entities or weak entities groups, and its frequency is greater than one, then the system uses the EHKB and linguistic rules to either discard the candidate noun or accept it as an entity (using human intervention).

\footnotetext{
${ }^{7}$ https://nlp.stanford.edu/software/CRF-NER.shtml
} 


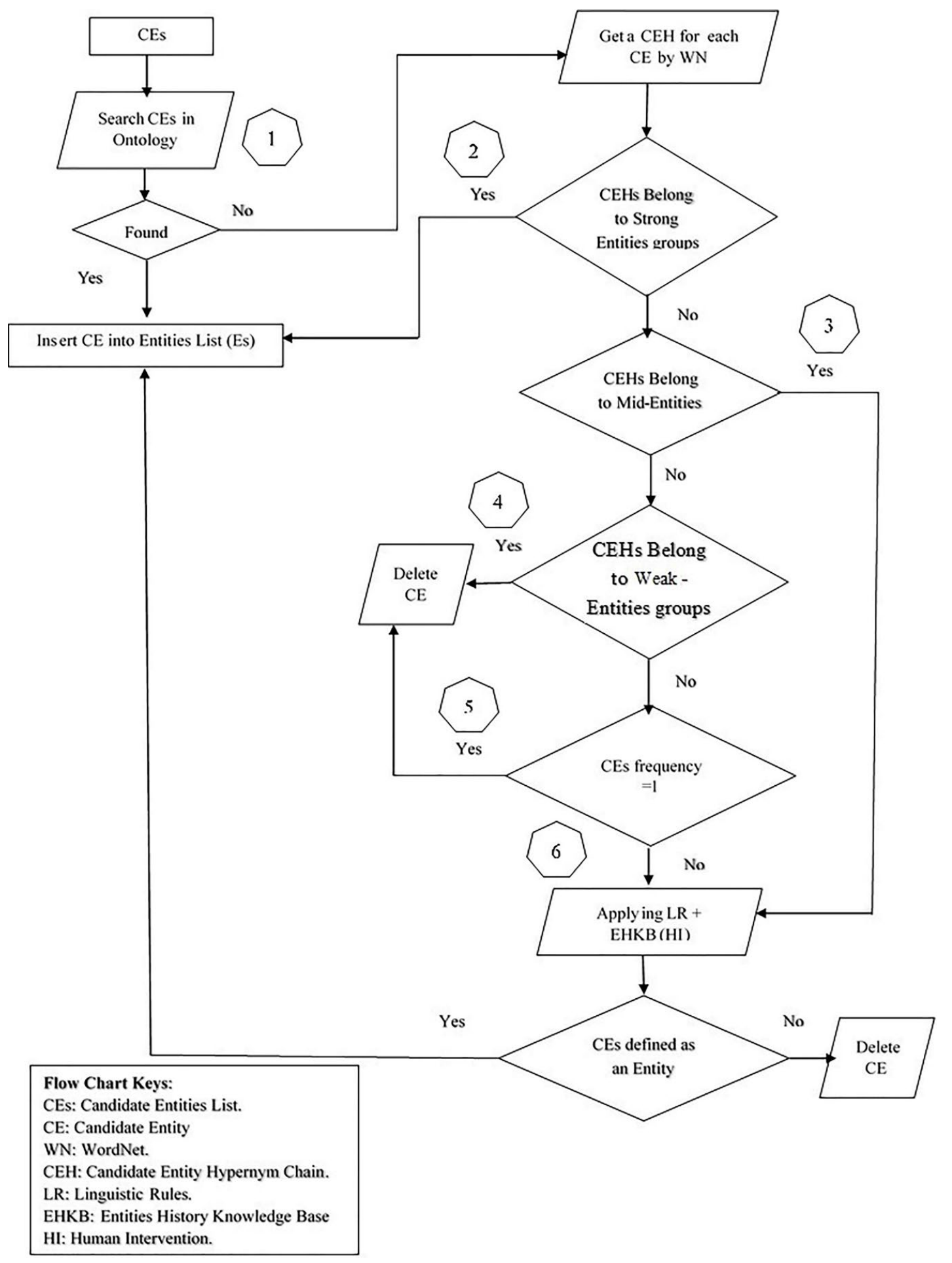

Fig. 4. Flow Chart of Entities Identification Stage

\subsection{Relationships Identification Stage}

Figure 5 demonstrates a flow chart for the relationships identification stage. This stage is divided into three sub-stages. The first sub-stage identifies relationships from the requirement specification text using Stanford typed dependencies. The second sub-stage identifies relationships from the entities list identified in the first stage. The third sub-stage involves human intervention to finalise the identified relationship and the resulting CM. A detailed analysis of these sub-stages follows. 


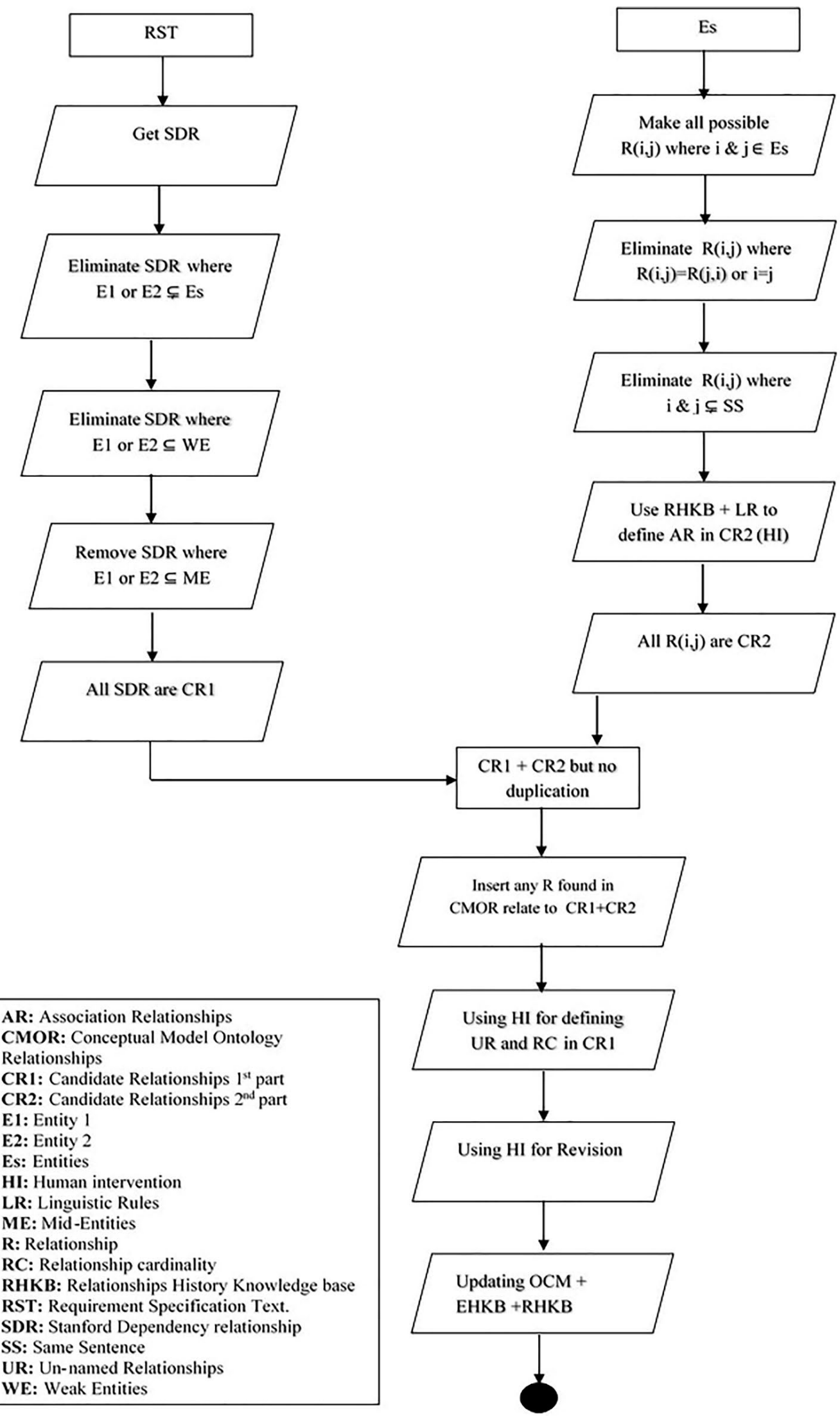

Fig. 5. Flow Chart of Relationships Identification Stage 


\subsubsection{Identifying relationships using Stanford typed dependencies}

The input for this sub-stage is the requirement specification text for a specific problem, and the outputs are candidate relationships defined by Stanford typed dependencies [27]. We employed Stanford dependencies as part of Stanford CoreNLP in this stage. Relationships are interactions between nouns and verbs. The nouns represent subjects and objects, the subject being a person or thing doing something and the object having something done to it. Stanford dependencies can deduce sentence subjects and sentence objects from the following list of relationships: nominal subject (nsubj), nominal subject passive (nsubjpass), clausal subject (csubj), passive clausal subject (csubjpass), direct object (dobj), indirect object (iobj) and preposition object (pobj).

Clausal subjects and passive clausal subjects represent sentence subjects in the form of a clause, and a clause cannot represent an entity. For example, in the sentence 'What Ali said makes sense', 'What Ali said' is a clausal subject. Therefore, clausal subjects are not mapped into entities. Similarly, an indirect object (iobj) is not useful because it represents a noun phrase stating a person or a thing which is influenced by the action of a transitive verb (typically as a recipient), but is not the primary object. For example, in 'She gives me a raise', the subject is 'she', the object is 'raise' and the action/verb is 'gives'. The indirect object is 'me'. A prepositional object (pobj) is equally unhelpful in defining a relationship because it modifies the noun rather than showing something done to the verb. For example, in 'A patient sat on the chair', the subject is 'A patient', the action/verb is 'sat' and 'on the chair' is a prepositional object.

A nominal subject (nsubj), however, is useful in defining a relationship because it shows who/what does the action. For example, in 'A school offers courses', 'A school' is nsubj for the action 'offers'. A direct object (dobj) is also useful in defining a relationship because it shows what is acted on by the verb. In the previous example, 'courses' is dobj for the action 'offers'. In addition, a nominal subject passive (nsubjpass) can be useful because it shows what/who does the action. A nominal subject passive (nsubjpass) is supported by an agent. An agent is the complement of a passive verb which is introduced by the preposition 'by' and does the action [27]. For example, in 'An invoice is paid by a customer', the nsubjpass is 'An invoice', while the agent is 'a customer'.

SACMES uses nsubj, dobj and nsubjpass to extract relationships from requirement specification text. The following example considers text which may form part of a requirement specification for a mall database: 'A customer buys many products. A customer is served by an employee.' Here, the Stanford dependency relationship of the first sentence is:

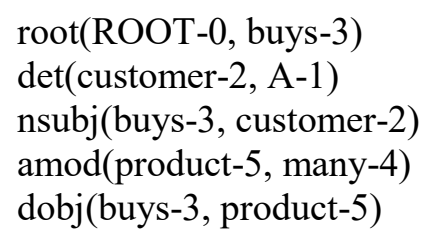

From nsubj(buys-3, customer-2), the action/verb 'buys' and the subject of the sentence 'customer' can be defined. From dobj(buys-3, product-5), the action/verb 'buys' and sentence object 'product' can be defined. Thus, from nsubj(buys-3, customer-2) and dobj(buys-3, product-5), the system can define the relationship whereby a customer buys a product in the following format: Buy (customer, product).

For the second sentence, the Stanford dependencies relationship is as follows:

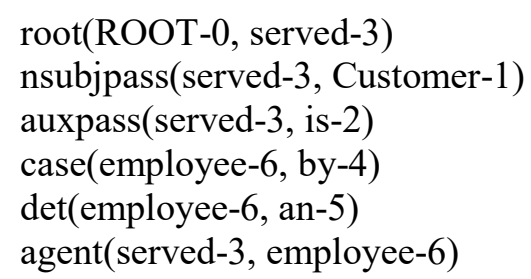

From the relationship nsubjpass(served-3, customer-1), the action/verb 'served' and the subject 'customer' can be defined. From the relationship agent(served-3, employee-6), the action/verb 'served' and agent 'employee' can be defined. Thus, from the relationship nsubjpass(served-3, customer-1) and 
the relationship agent(served-3, employee-6), the system can define the relationship whereby an employee serves a customer in the following format: Served (employee, customer).

As shown in Fig. 5, the following steps are applied in this sub-stage:

1. The system employs Stanford dependencies to extract relationships from requirement specification text.

2. The system eliminates any relationship involving a subject or object that is not included in the entities list defined in the entities identification stage.

3. The system eliminates any relationship that includes entities with a hypernym chain matching the weak or mid-entities groups. WordNet is incorporated to achieve this mission. The remaining relationships are called Candidate Relationships $1^{\text {st }}$ part (CR1).

\subsubsection{Identifying relationships from entities}

The input of this stage is the entities list defined in the entities identification stage. As shown in Fig. 5, the following steps are applied:

1. The system uses the entities list to determine all possible binary relationships between the entities. For example, if the three entities defined from the entities identification stage are 'customer', 'product' and 'employee', the possible binary relationships between these entities are:

$$
\begin{aligned}
& \text { (customer, customer) } \\
& \text { (customer, product) } \\
& \text { (customer, employee) } \\
& \text { (product, product) } \\
& \text { (product, employee) } \\
& \text { (product, customer) } \\
& \text { (employee, employee) } \\
& \text { (employee, product) } \\
& \text { (employee, customer) }
\end{aligned}
$$

2. Reversing the order of terms should not produce a distinct relationship. For example, the relationships (customer, product) and (product, customer) match this condition. After eliminating such redundancies in the list of relationships, the list is updated as follows.

$$
\begin{aligned}
& \text { (customer, customer) } \\
& \text { (customer, product) } \\
& \text { (customer, employee) } \\
& \text { (product, product) } \\
& \text { (product, employee) } \\
& \text { (employee, employee) }
\end{aligned}
$$

3. Similarly, entities should not have relationships to themselves, as with (customer, customer), for example. After eliminating the relationships that meet this condition, the relationships list is updated as follows.

$$
\begin{aligned}
& \text { (customer, product) } \\
& \text { (customer, employee) } \\
& \text { (product, employee) }
\end{aligned}
$$

4. The process eliminates any relationship that does not have a first and second entity both mentioned in one sentence within a requirement specification text. The entities that are mentioned in the same sentence may have a relationship between them. However, the system then uses human intervention to revise the result by adding any missing relationships and removing any incorrect relationships. 
5. The system requires users to use the Relationships History Knowledge Base (RHKB) and needto-know rule to define the associations within those relationships that remain after the above filtering steps.

6. The remaining relationships are are called Candidate Relationships $2^{\text {nd }}$ part (CR2).

\subsubsection{Final Stage: Human Intervention and Updating OCM and UHKB}

The inputs of this stage are CR1 and CR2. Before the stage is started, the system searches the OCM for relationships identified in CR1 and CR2, and adds them to the relationships list. The system then asks the user to define the cardinality of relationships, and give appropriate names to unnamed relationships. The user is also given the opportunity to review the results of the process in order to add or remove entities or relationships, as necessary. The user can then print a report containing a list of entities and list of relationships defined for the problem. This is the final step in SACMES, where the user is directly involved. The system also updates the OCM by adding these entities and relationships into the ontology, as well as updating the UHKB by saving the user's behaviour into the database.

\section{Evaluation}

\subsection{First Experiment: Performance Improvement using SACMES}

In this section, an empirical evaluation is conducted to confirm that the performance of designers will be improved when using SACMES, in comparison to their manual performance. A test set of twenty case studies has been established, the case studies having been collected from authentic resources including database textbooks and $\mathrm{PhD}$ theses. The test set is divided into easy problems and harder problems, with ten case studies in each sub-set. Clearly, the easy problems are less complex than the harder problems, and the use of both types is intended to demonstrate that the system can deal with both easy and complex cases. Each case study has a set of model answers, which includes entities, relationships and cardinalities of relationships. Some cases were found with their model answers, while other model answers (12 case studies) were created by an expert designer ${ }^{8}$ and evaluated by the second author. We consider as expert designers those that have considerable experience in building CMs either in industry or in academia (having completed doctoral studies and/or serving in lecturing positions). Twenty subjects participated in the experiment, all of whom are novice designers. The size of the test set as well as the number of participating subjects are considered more than adequate compared to similar studies. For example, Elbendak's study [28] used a test corpus of eight case studies and nine participating subjects to measure the performance of the Class-Gen tool that maps natural language text into classes. Furthermore, the performance of Taxonomic Class Modelling (TCM) [29] was tested using eight case studies.

While expert designers are more capable and skilled at translating natural language specifications into CMs, novice designers are less skilled at this task. We wished to observe how SACMES would support novice designers in producing CMs, and it was for this reason that we chose to include novice designers as subjects for the experiment. Each subject was requested to fill in a questionnaire. This questionnaire helped us to determine the extent to which the subjects were suitable for participation in the experiment, to discover their background with regard to conceptual model creation, and to receive feedback regarding their use of SACMES. All the subjects are undergraduate and postgraduate students in the Computer Science Department of the Computing and Engineering School at the University of Huddersfield in the United Kingdom. None of them have extensive experience in the creation of conceptual models, though the majority have studied CMs during their courses. The subjects were divided into two groups, namely, Group One and Group Two, with ten subjects in each group. Each subject provided four answers for four different case studies from the test set, two from the easy group and two from the harder group. One easy and one harder case study had to be solved manually, while

\footnotetext{
${ }^{8}$ https://www.linkedin.com/in/hadeel-jazzaa-b6375363
} 
the other two had to be solved using SACMES. Table 3 illustrates the activities undertaken by the subjects during the experiment.

All answers were compared with the model answers in order to determine the extent to which the subjects' performance was affected by using SACMES. Answers provided by subjects with the help of the system are called system answers, while those provided without using the system are called manual answers. The subjects' answers are classified into three classes, which are Correct (COR), Incorrect (INC) and Missed (MISS). An answer is classified as correct when it is found as both a model answer and a system answer, or a model answer and a manual answer. An answer is classified as incorrect when it is found in the system answer or the manual answer but is not included in the model answer. An answer is classified as missed when it is included in the model answer but not found in the system answer or manual answer. Recall $\left(\frac{C O R}{C O R+M I S S}\right)$ and precision $\left(\frac{C O R}{C O R+I N C}\right)$ are used to evaluate the extent to which system answers and manual answers match model answers. Recall and precision were originally developed for use in evaluating information retrieval systems, but are now widely used to evaluate the performance of information extraction systems [28].

Table 3. Activities of subjects in first experiment

\begin{tabular}{|l|l|l|l|l|}
\hline Subject & Problem & Problem & \multicolumn{1}{|c|}{ Problem } & Problem \\
\hline S1 & E1WO & E2W & H1WO & H2W \\
\hline S2 & E1W & E2WO & H1W & H2WO \\
\hline S3 & E3WO & E4W & H3WO & H4W \\
\hline S4 & E3W & E4WO & H3W & H4WO \\
\hline S5 & E5WO & E6W & H5WO & H6W \\
\hline S6 & E5W & E6WO & H5W & H6WO \\
\hline S7 & E7WO & E8W & H7WO & H8W \\
\hline S8 & E7W & E8WO & H7W & H8WO \\
\hline S9 & E9WO & E10W & H9WO & H10W \\
\hline S10 & E9W & E10WO & H9W & H10WO \\
\hline $\begin{array}{l}\text { E: Easy case study, H: Harder case study } \\
\text { W: With using SACMES, WO: Without using SACMES }\end{array}$ \\
\hline
\end{tabular}

\subsubsection{First Group Results}

Table 4. Performance Results for First Group (Manual before SACMES)

\begin{tabular}{|c|c|c|}
\hline \multicolumn{3}{|c|}{ Entities Extraction } \\
\hline & Recall (Average) & Precision (Average) \\
\hline Manual & $71.50 \%$ & $81.29 \%$ \\
\hline SACMES & $84.46 \%$ & $87.41 \%$ \\
\hline \multicolumn{3}{|c|}{ Relationships Extraction } \\
\hline & Recall (Average) & Precision (Average) \\
\hline Manual & $41.47 \%$ & $48.06 \%$ \\
\hline SACMES & $64.03 \%$ & $62.51 \%$ \\
\hline \multicolumn{3}{|c|}{ Cardinality Extraction } \\
\hline & Recall (Average) & Precision (Average) \\
\hline Manual & $32.43 \%$ & $28.75 \%$ \\
\hline SACMES & $42.77 \%$ & $30.70 \%$ \\
\hline
\end{tabular}

Model answers were compared with the system answers and manual answers and the average results across all subjects are shown in Table 4. From the results, it can be concluded that novice designers' performance in entities extraction improved across the board when using SACMES. This result supports the hypothesis that the performance of novice designers improves when they use SACMES comparing to their manual performance. Note that as the system learns from the natural language text that it processes, its performance of the system will improve as it processes more case studies (more on this 
in the second experiment). Therefore, it is encouraging that, even though the system had so far only processed a few case studies, the average performance of the subjects still improved when using it.

\subsubsection{Second Group Results}

Before the subjects in the second group started, the information learnt by the system and stored in the CMO and UHKB database was deleted so that it would not affect these subjects' performance. The steps performed by the second group of subjects were the same as those followed by the first group. The only difference was that while the subjects in the first group gave their handcrafted answers first, before using the system, the subjects in the second group started by using the system and then gave their handcrafted answers afterwards. The reason behind this was to ensure that the improvement in performance shown by the first group was not because they were doing the job of creating CMs for the second time, having learnt from the first time. If the performance of subjects in the second group is also improved by using the system, despite starting by using it, then it can be presumed that the subjects' improvement was purely due to their use of the system.

Table 5. Performance Results for Second Group (SACMES before Manual)

\begin{tabular}{|c|c|c|}
\hline \multicolumn{3}{|c|}{ Entities Extraction } \\
\hline & Recall (Average) & Precision (Average) \\
\hline Manual & $78.95 \%$ & $85.45 \%$ \\
\hline SACMES & $88.95 \%$ & $84.68 \%$ \\
\hline \multicolumn{3}{|c|}{ Relationships Extraction } \\
\hline & Recall (Average) & Precision (Average) \\
\hline Manual & $60.81 \%$ & $62.50 \%$ \\
\hline SACMES & $70.85 \%$ & $68.66 \%$ \\
\hline \multicolumn{3}{|c|}{ Cardinality Extraction } \\
\hline & Recall (Average) & Precision (Average) \\
\hline Manual & $55.88 \%$ & $52.20 \%$ \\
\hline SACMES & $67.04 \%$ & $56.53 \%$ \\
\hline
\end{tabular}

Results are shown in Table 5. It should be noted that comparison to model answers is rather strict: many of the answers provided by the subjects when using the system could have been correct answers, but were classified as incorrect because they were not identified in the model answers. Alternatively, a set of performance criteria could be set by experts and used to evaluate answers provided with and without using SACMES.

\subsection{Second Experiment: Learning Ability of SACMES}

With the second experiment, we attempt to provide evidence that the knowledge and information stored by SACMES helps to improve the performance of the system and minimise human intervention. In order to provide evidence of this, it was necessary to train the system to learn and then measure the performance of the system after it had learnt. To train a system to learn, a training set must be developed. For this purpose, a collection of fifty case studies was used, taken from authentic resources such as database textbooks and $\mathrm{PhD}$ theses. The training set was divided into ten groups, each with five case studies. A different collection of five case studies was used as test set. Two of these case studies were found with their answers, while model answers for the other three were provided by the same experts as in the first experiment. Before starting this experiment, we considered the subjects who would participate in the experiment. The initial intention was to find fifty students, one for each case study, as subjects to train the system, but not enough students expressed interest. As an alternative, it was decided that the first author, who has some experience in the creation of CMs, having studied this during his undergraduate course, would be eligible to participate in the study. He therefore played the role of designer for all case studies and performed the tasks required to train the system. Note that this is not a source of bias for this experiment, as the purpose is to investigate whether and to what extent SACMES 
can learn from available knowledge, regardless of the quality of that knowledge and how it may have been collected.

Since SACMES includes human intervention, the output is always dependent on the user and on previous runs that have populated the CMO and UHKB. In this experiment, however, it was important for the output of the system to rely only the knowledge stored in SACMES. Therefore, we needed to use two different versions of SACMES. The first version, depicted in Fig. 1, was used by the first author to train the system. The second version, called Knowledge-Based Conceptual Model Extraction System (KBCMES), differs from the first in that it does not require human intervention and does not store the outputs of the system in the CMO and UHKB, as shown in Fig. 6. The results of this system are only dependent on its knowledge, rather than the system users. Furthermore, the outputs are not stored in $\mathrm{CMO}$ or UHKB, to ensure that no information is added into the system apart from that which is added during each training stage. KBCMES was used to extract CMs for the test set after finishing each of the training stages.

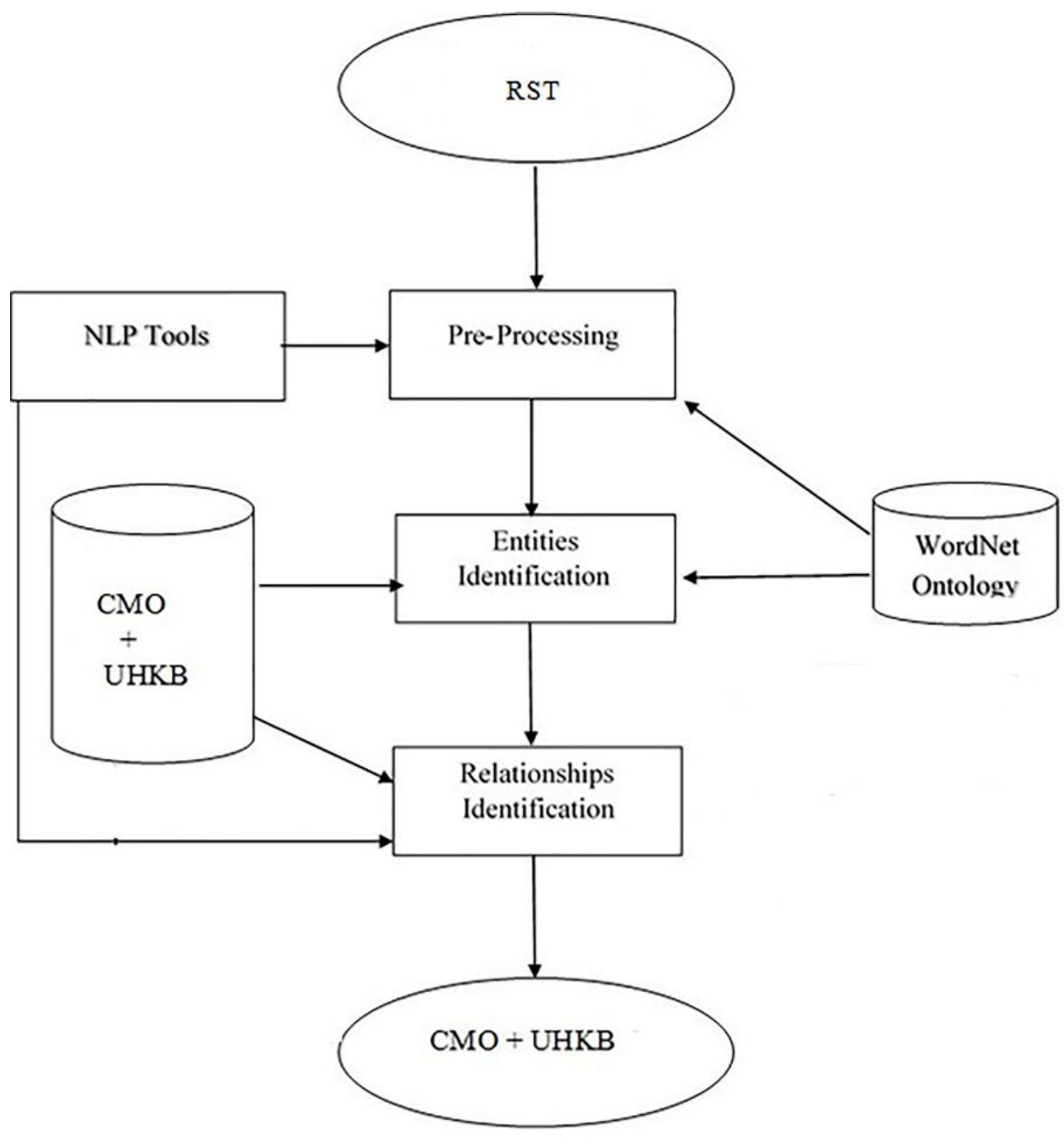

Fig. 6. System Architecture for KBCMES

Before SACMES was trained on the training set, KBCMES was used to obtain CMs for the test set. The recall and precision for each case study within the test set were recorded. Next, SACMES was used to train the CMO and UHKB by using group number one of the training set, which includes five case studies. Fresh copies of the CMO and RHKB were then used and trained on ten case studies from the training set. This process was repeated until we obtained ten copies of the CMO and RHKB, the first copy trained on five case studies, the second copy trained on ten case studies, and so on, with the tenth 
copy trained on all fifty case studies. Each copy of the CMO and RHKB was then fed to KBCMES separately to obtain CMs for the test set, and the recall and precision for each case study were recorded.

\subsubsection{Results and Discussion}

Table 6 presents the results obtained by using KBCMES to extract CMs for case studies within the test set before any training was performed, when there was no information in the CMO or UHKB database. For already defined entities, relationships and cardinalities, as well as unrecognised entities and relationships in each dataset, we count correct and incorrect answers returned by KBCMES. In most of the cases, the system was unable to provide any answer. This should be expected since no training has been performed. Table 7 presents a results summary obtained from using KBCMES integrated with a CMO and UHKB database trained on all fifty case studies.

Table 6. Results Obtained for Test Set from KBCMES before Training

\begin{tabular}{|c|c|c|c|c|c|c|}
\hline \multicolumn{7}{|c|}{ Unrecognised Entities } \\
\hline Case study & Total & $\begin{array}{l}\text { Correct } \\
\text { answer }\end{array}$ & $\begin{array}{l}\text { Incorrect } \\
\text { answer }\end{array}$ & $\begin{array}{l}\text { No } \\
\text { answer }\end{array}$ & Recall & Precision \\
\hline VedMed & 13 & 0 & 0 & 13 & $0 \%$ & $0 \%$ \\
\hline DreamHome & 14 & 0 & 0 & 14 & $0 \%$ & $0 \%$ \\
\hline Airline & 18 & 0 & 0 & 18 & $0 \%$ & $0 \%$ \\
\hline Florida Mall & 9 & 0 & 0 & 9 & $0 \%$ & $0 \%$ \\
\hline Coca Cola & 14 & 0 & 0 & 14 & $0 \%$ & $0 \%$ \\
\hline \multirow{2}{*}{\multicolumn{7}{|c|}{ Unrecognised Relationships }} \\
\hline & & & & & & \\
\hline Case study & Total & $\begin{array}{l}\text { Correct } \\
\text { answer }\end{array}$ & $\begin{array}{l}\text { Incorrect } \\
\text { answer }\end{array}$ & $\begin{array}{l}\text { No } \\
\text { answer }\end{array}$ & Recall & Precision \\
\hline VedMed & 61 & 0 & 0 & 61 & $0 \%$ & $0 \%$ \\
\hline DreamHome & 121 & 0 & 0 & 121 & $0 \%$ & $0 \%$ \\
\hline Airline & 58 & 0 & 0 & 58 & $0 \%$ & $0 \%$ \\
\hline Florida Mall & 70 & 0 & 0 & 70 & $0 \%$ & $0 \%$ \\
\hline Coca Cola & 111 & 0 & 0 & 111 & $0 \%$ & $0 \%$ \\
\hline \multirow{2}{*}{\multicolumn{7}{|c|}{ Entities }} \\
\hline & & & & & & \\
\hline Case study & Total & $\begin{array}{l}\text { Correct } \\
\text { answer }\end{array}$ & $\begin{array}{l}\text { Incorrect } \\
\text { answer }\end{array}$ & $\begin{array}{l}\text { No } \\
\text { answer }\end{array}$ & Recall & Precision \\
\hline VedMed & 4 & 0 & 0 & 4 & $0 \%$ & $0 \%$ \\
\hline DreamHome & 8 & 0 & 0 & 8 & $0 \%$ & $0 \%$ \\
\hline Airline & 7 & 2 & 0 & 5 & $28.57 \%$ & $100 \%$ \\
\hline Florida Mall & 7 & 0 & 0 & 7 & $0 \%$ & $0 \%$ \\
\hline Coca Cola & 9 & 0 & 0 & 9 & $0 \%$ & $0 \%$ \\
\hline & & & & Average & $5.71 \%$ & $20 \%$ \\
\hline \multicolumn{7}{|c|}{ Relationships } \\
\hline Case study & Total & $\begin{array}{l}\text { Correct } \\
\text { answer }\end{array}$ & $\begin{array}{l}\text { Incorrect } \\
\text { answer }\end{array}$ & $\begin{array}{l}\text { No } \\
\text { answer }\end{array}$ & Recall & Precision \\
\hline VedMed & 3 & 0 & 0 & 3 & $0 \%$ & $0 \%$ \\
\hline DreamHome & 10 & 0 & 0 & 10 & $0 \%$ & $0 \%$ \\
\hline Airline & 7 & 0 & 0 & 7 & $0 \%$ & $0 \%$ \\
\hline Florida Mall & 9 & 0 & 0 & 9 & $0 \%$ & $0 \%$ \\
\hline Coca Cola & 12 & 0 & 0 & 12 & $0 \%$ & $0 \%$ \\
\hline \multirow{2}{*}{\multicolumn{7}{|c|}{ Cardinalities }} \\
\hline & & & & & & \\
\hline Case study & Total & $\begin{array}{l}\text { Correct } \\
\text { answer }\end{array}$ & $\begin{array}{l}\text { Incorrect } \\
\text { answer }\end{array}$ & $\begin{array}{l}\text { No } \\
\text { answer }\end{array}$ & Recall & Precision \\
\hline VedMed & 3 & 0 & 1 & 2 & $0 \%$ & $0 \%$ \\
\hline
\end{tabular}




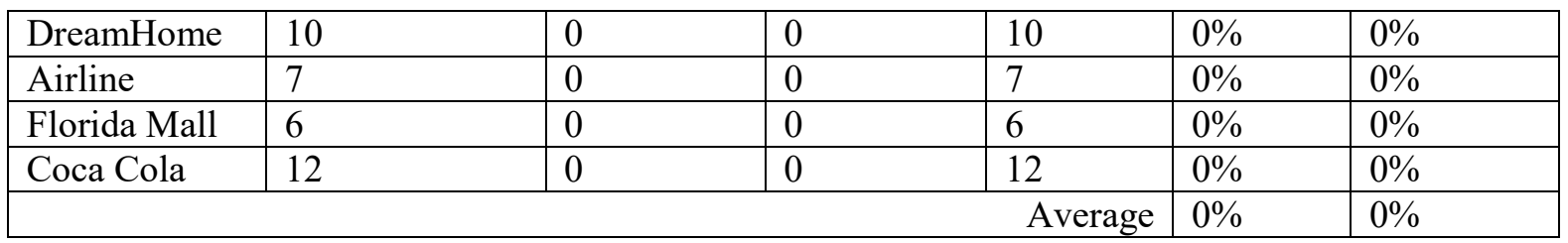

Table 7. Results Obtained for Test Set from KBCMES after Training

\begin{tabular}{|c|c|c|c|c|c|c|}
\hline \multicolumn{7}{|c|}{ Unrecognised Entities } \\
\hline Case study & Total & $\begin{array}{l}\text { Correct } \\
\text { answer }\end{array}$ & $\begin{array}{l}\text { Incorrect } \\
\text { answer }\end{array}$ & $\begin{array}{l}\text { No } \\
\text { answer }\end{array}$ & Recall & Precision \\
\hline VedMed & 13 & 8 & 0 & 5 & $61.53 \%$ & $100 \%$ \\
\hline DreamHome & 13 & 4 & 0 & 9 & $30.76 \%$ & $100 \%$ \\
\hline Airline & 18 & 4 & 0 & 14 & $22.22 \%$ & $100 \%$ \\
\hline Florida Mall & 9 & 2 & 0 & 7 & $22.22 \%$ & $100 \%$ \\
\hline Coca Cola & 14 & 5 & 0 & 9 & $35.71 \%$ & $100 \%$ \\
\hline \multirow{2}{*}{\multicolumn{7}{|c|}{$\begin{array}{l}\text { Average } \\
\text { Unrecognised Relationships }\end{array}$}} \\
\hline & & & & & & \\
\hline Case study & Total & $\begin{array}{l}\text { Correct } \\
\text { answer }\end{array}$ & $\begin{array}{l}\text { Incorrect } \\
\text { answer }\end{array}$ & $\begin{array}{l}\text { No } \\
\text { answer }\end{array}$ & Recall & Precision \\
\hline VedMed & 61 & 15 & 1 & 45 & $25 \%$ & $93.75 \%$ \\
\hline DreamHome & 127 & 22 & 2 & 103 & $17.6 \%$ & $91.66 \%$ \\
\hline Airline & 58 & 14 & 1 & 43 & $24.56 \%$ & $93.33 \%$ \\
\hline Florida Mall & 70 & 9 & 3 & 58 & $13.43 \%$ & $75 \%$ \\
\hline Coca Cola & 111 & 33 & 3 & 75 & $30.55 \%$ & $91.66 \%$ \\
\hline \multirow{2}{*}{\multicolumn{7}{|c|}{ Average }} \\
\hline & & & & & & \\
\hline Case study & Total & $\begin{array}{l}\text { Correct } \\
\text { answer }\end{array}$ & $\begin{array}{l}\text { Incorrect } \\
\text { answer }\end{array}$ & $\begin{array}{l}\text { No } \\
\text { answer }\end{array}$ & Recall & Precision \\
\hline VedMed & 4 & 1 & 1 & 2 & $25 \%$ & $50 \%$ \\
\hline DreamHome & 8 & 1 & 2 & 5 & $12 . \%$ & $33.33 \%$ \\
\hline Airline & 7 & 3 & 1 & 3 & $42.85 \%$ & $75 \%$ \\
\hline Florida Mall & 7 & 3 & 2 & 2 & $42.85 \%$ & $60 \%$ \\
\hline Coca Cola & 9 & 7 & 0 & 2 & $77.77 \%$ & $100 \%$ \\
\hline \multirow{2}{*}{\multicolumn{7}{|c|}{ Average }} \\
\hline & & & & & & \\
\hline Case study & Total & $\begin{array}{l}\text { Correct } \\
\text { answer }\end{array}$ & $\begin{array}{l}\text { Incorrect } \\
\text { answer }\end{array}$ & $\begin{array}{l}\text { No } \\
\text { answer }\end{array}$ & Recall & Precision \\
\hline VedMed & 3 & 0 & 1 & 2 & $0 \%$ & $0 \%$ \\
\hline DreamHome & 10 & 0 & 2 & 8 & $0 \%$ & $0 \%$ \\
\hline Airline & 7 & 0 & 2 & 5 & $0 \%$ & $0 \%$ \\
\hline Florida Mall & 9 & 1 & 3 & 5 & $11.11 \%$ & $25 \%$ \\
\hline Coca Cola & 12 & 5 & 1 & 6 & $41.66 \%$ & $83.33 \%$ \\
\hline \multirow{2}{*}{\multicolumn{7}{|c|}{ Average }} \\
\hline & & & & & & \\
\hline Case study & Total & $\begin{array}{l}\text { Correct } \\
\text { answer }\end{array}$ & $\begin{array}{l}\text { Incorrect } \\
\text { answer }\end{array}$ & $\begin{array}{l}\text { No } \\
\text { answer }\end{array}$ & Recall & Precision \\
\hline VedMed & 3 & 0 & 1 & 2 & $0 \%$ & $0 \%$ \\
\hline DreamHome & 10 & 0 & 2 & 8 & $0 \%$ & $0 \%$ \\
\hline Airline & 7 & 0 & 1 & 6 & $0 \%$ & $0 \%$ \\
\hline Florida Mall & 6 & 1 & 3 & 2 & $16.66 \%$ & $25 \%$ \\
\hline Coca Cola & 12 & 4 & 2 & 6 & $36.36 \%$ & $66.66 \%$ \\
\hline \multicolumn{5}{|r|}{ Average } & $10.60 \%$ & $18.33 \%$ \\
\hline
\end{tabular}


Results in Table 7 show that there is improvement across the board and system performance has improved and its need for human intervention has been reduced in comparison with the result for the same section in Table 6. It can be seen that results on average are better for entities than relationships and cardinalities. This can be attributed to the technologies behind extraction and the linguistic differences between entities and relationships. Entities extraction relied on WordNet which divides nouns in strong, mid- and weak entities. SACMES considers only strong entities to be entities, hence WordNet's categorisation seems to be accurate. On the other hand, relationships extraction relies on Stanford typed dependencies. For simple sentences, such as "Students study modules", extraction is straightforward. However, since there are different ways of expressing relationships other than simple subject-verb-object sentences, Stanford typed dependencies are less successful in capturing these. For cardinalities, results point out that the training set is insufficient and further improvement would depend on larger numbers of use cases. The differences in improvement from one case study to another can be partly attributed to differences in syntax, structure and expression.

Table 8. Results in Relation to the Size of Training Set

\begin{tabular}{|l|l|l|l|l|l|l|l|l|l|l|l|}
\hline \multicolumn{1}{|c|}{ Unrecognised Entities } \\
\hline & Count of case studies used in training \\
\cline { 2 - 12 } & 0 & 5 & 10 & 15 & 20 & 25 & 30 & 35 & 40 & 45 & 50 \\
\hline Recall (\%) & 0 & 7.66 & 8.83 & 23.61 & 25.15 & 25.15 & 26.57 & 26.57 & 28.11 & 28.11 & 34.48 \\
\hline Precision (\%) & 0 & 100 & 100 & 100 & 100 & 100 & 100 & 100 & 100 & 100 & 100 \\
\hline \multicolumn{8}{|c|}{ Unrecognised Relationships } \\
\hline \\
\hline
\end{tabular}

Table 8 demonstrates how recall and precision scores change, as more case studies are added to the training set. For unrecognised entities and relationships, there is continuous improvement by the addition of more case studies. In the case of entities precision is constant, with an insignificant drop when 15 case studies are used. For recall, there is a fluctuation around 25 case studies, but results seem to increase steadily from 35 case studies onwards. Results for relationships and cardinalities with regard to recall are similar: there is a slight decrease for recall at 20 case studies after which there is steady increase. Precision for relationships and cardinalities drops at 15 case studies but eventually settles at slightly higher values.

These results show that the system learns from the natural language specifications processed by users, and uses the knowledge stored from these specifications to improve the extraction of ERDs from specifications that will be processed in the future. As a result, the system's performance is enhanced. The observed fluctuations that may be attributed to the characteristics of some case studies seem to be smoothed over as we reach the maximum available case studies. This is positive proof that the information stored by SACMES in the CMO and UHKB can help the system improve its performance, minimise the need for human intervention and enable it to produce more relevant information to advise users in the creation of CMs. It should also be noted that the training set contains many domains that 
are completely different from the domains included in the test set. Despite there being no systematic relationship between the domains included in the training set and test set, system performance is still improved. Higher performance should be expected in cases where both the training set and test set belong to the same domain, or in cases where the training is significantly larger, containing hundreds of natural language specifications.

\section{Conclusion and Future Work}

The purpose of this research is to improve the creation of CMs by producing a tool to assist designers in this process. We made a contribution to knowledge by developing a system for extracting CMs from natural language text for domain independent. The developed tool that supports the system learns from the natural language texts that it processes and stores what has been learnt in its knowledge base to update it. The information that is stored in the knowledge base helps the tool to minimise human intervention and to improve its performance. Human intervention is mainly required to resolve ambiguities and cases of missing information with regard to entities and relationships, as well as to review the final result. Experiments were conducted on the use of SACMES by novice designers, and the results showed improvement in the designers' performance when they used the system compared to when not using it. To prove that information stored by the system will be useful in improving the system's performance. The results obtained after training the system on a set of fifty case studies showed that the performance of the system improved by increasing the number of case studies used to train it.

In the future, we intend to explore ways of further reducing human intervention by: (1) implementing methods to validate entities and relationships before they are added to the knowledge base and preventing invalid ones from being stored; and (2) exploiting additional machine learning techniques such as Naïve Bayes classifiers to improve accuracy in identifying entities and relationships in natural language text. Future work could also be undertaken to develop retrieval techniques that go beyond spelling matching to ensure that the system remains efficient even when the information stored increases considerably. Also, SACMES is currently a local, single-user system and we intend to explore multi-user scenarios by providing the system to multiple participants in different locations as a servicebased application [30-32]. Finally, to reduce sources of potential bias in future experiments, we intend to include a panel of expert designers for the creation of model answers, in case studies where these are unavailable.

\section{Acknowledgment}

This research did not receive any specific grant from funding agencies in the public, commercial, or not-for-profit sectors.

\section{References}

[1] Thalheim, B. (2000). Entity-Relationship Modelling: Foundations of Database Technology. New York: Springer-Verlag, Inc.

[2] Dullea, J., Song, I.-Y., \& Lamprou, I. (2003). An analysis of structural validity in entity-relationship modelling. Data \& Knowledge Engineering, 47(2), 167-205. doi: https://doi.org/10.1016/S0169023X(03)00049-1.

[3] Chen, P. P. S. (1976). The entity-relationship model-toward a unified view of data. ACM Trans. Database Syst., 1(1), 9-36. doi: 10.1145/320434.320440.

[4] Neill, C. J., \& Laplante, P. A. (2003). Requirement engineering: the state of the practice. IEEE Software, 20(6), 40-45.

[5] Boehm, B. W. (1981). Software engineering economics (Prentice-Hall Advances in Computing Science and Technology Series). Englewood Cliffs: Prentice-Hall. 
[6] Song, I.-Y., Zhu, Y., Ceong, H., \& Thonggoom, O. (2015). Methodologies for Semi-automated Conceptual Data Modelling from Requirements. In $34^{\text {th }}$ International Conference on Conceptual Modelling, Stockholm, Sweden, 18-31.

[7] Omer, M. A. M., \& Wilson, D. (2016, October). New rules for deriving formal models from text. In 2016 International Conference for Students on Applied Engineering (ICSAE) (pp. 328-333). IEEE.

[8] Šuman, S., Jakupović, A., \& Kuljanac, F. G. (2016). Knowledge-Based Systems for Data Modelling. International Journal of Enterprise Information Systems (IJEIS), 12(2), 1-13.

[9] Thonggoom, O. (2011). Semi-automatic Conceptual Data Modelling Using Entity and Relationship Instance Repositories ( $\mathrm{PhD}$ thesis). Drexel University, Philadelphia, PA, USA.

[10] Harmain, H. M., \& Gaizauskas, R. (2003). CM-Builder: A Natural Language-Based CASE Tool for Object-Oriented Analysis. Automated Software Engineering, 10(2), 157-181. doi: 10.1023/A:1022916028950.

[11] Chen, P. P. S. (1983). English sentence structure and entity-relationship diagrams. Information Sciences, 29(2), 127-149.

[12] Omar, N., Hanna, J. R. P, \& McKevitt, P. (2004). Heuristic-based entity-relationship modelling through natural language processing. In Proc. of the 15th Artificial Intelligence and Cognitive Science Conference (AICS), Galway-Mayo Institute of Technology (GMIT), Castlebar, Ireland, 302-313.

[13] Wohed, P. (2000). Conceptual patterns for reuse in information systems analysis. In International Conference on Advanced Information Systems Engineering. Springer, Berlin, Heidelberg, 157-175

[14] Purao, S. (1998). APSARA: a tool to automate system design via intelligent pattern retrieval and synthesis. ACM SIGMIS Database, 29(4), 45-57.

[15] Storey, V. C., Chiang, R. H., Dey, D., Goldstein, R. C., \& Sudaresan, S. (1997). Database design with common sense business reasoning and learning. ACM Transactions on Database Systems (TODS), 22(4), 471-512.

[16] Paek, Y.-K., Seo, J., \& Kim, G.-C. (1996). An expert system with case-based reasoning for database schema design. Decision Support Systems, 18(1), 83-95.

[17] Sugumaran, V., \& Storey, V. C. (2006). The role of domain ontologies in database design: An ontology management and conceptual modelling environment. ACM Trans. Database Syst., 31(3), 1064-1094. doi: 10.1145/1166074.1166083.

[18] Herchi, H. \& Abdessalem, W. B. (2012). From user requirements to UML class diagram. In International Conference on Computer Related Knowledge, Sousse, Tunisia. Retrieved from http://arxiv.org/abs/1211.0713.

[19] Sugumaran, V., \& Storey, V. C. (2002). Ontologies for conceptual modelling: their creation, use, and management. Data \& Knowledge Engineering, 42(3), 251-271. doi: https://doi.org/10.1016/S0169023X(02)00048-4.

[20] Thonggoom, O., Song, I.-Y., \& An, Y. (2011). EIPW: A Knowledge-Based Database Modelling Tool. In C. Salinesi \& O. Pastor (Eds), Advanced Information Systems Engineering Workshops. CAiSE 2011. Lecture Notes in Business Information Processing, 83. Berlin, Heidelberg: Springer.

[21]Al-Btoush, A. A.-S. (2015). Extracting Entity Relationship Diagram (ERD) from English Sentences. International Journal of Database Theory and Application, 8(2), 235-244.

[22] Ben Abdessalem Karaa, W., Ben Azzouz, Z., Singh, A., Dey, N., Ashour, A., \& Ben Ghazala, H. (2016). Automatic builder of class diagram (ABCD): an application of UML generation from functional requirements. Software: Practice and Experience, 46(11), 1443-1458.

[23] Manning, C. D., Surdeanu, M., Bauer, J., Finkel, J. R., Bethard, S., \& McClosky, D. (2014). The Stanford CoreNLP Natural Language Processing toolkit. In Proceedings of 52nd Annual Meeting of the Association for Computational Linguistics: System Demonstrations, Baltimore, Maryland USA, 55-60. [24] Toutanova, K., Klein, D., Manning, C. D., \& Singer, Y. (2003). Feature-rich part-of-speech tagging with a cyclic dependency network. In Proceedings of the 2003 Conference of the North American Chapter of the Association for Computational Linguistics on Human Language Technology, Edmonton, Canada, 173-180.

[25] Btoush, E. S., \& Hammad, M. M. (2015). Generating ER Diagrams from Requirement Specifications Based On Natural Language Processing. International Journal of Database Theory and Application, 8(2), 61-71. 
[26] Tjong Kim Sang, E. F., \& De Meulder, F. (2003). Introduction to the CoNLL-2003 shared task: Language-independent named entity recognition. In Proceedings of the seventh conference on Natural language learning at HLT-NAACL 2003, Edmonton Canada-Volume 4, 142-147.

[27] De Marneffe, M.-C., \& Manning, C. D. (2008). Stanford typed dependencies manual, Stanford University. Retrieved from https://nlp.stanford.edu/software/dependencies_manual.pdf

[28] Elbendak, M. E. (2011). Requirements-driven Automatic Generation of Class Models (PhD thesis). Northumbria Univeristy, Newcastle upon Tyne.

[29] Song, I.-Y., Yano, K., Trujillo, J., \& Luján-Mora, S. (2004). A taxonomic class modelling methodology for object-oriented analysis. IGI Global, 216-240. doi:10.4018/978-1-59140-3753.ch011.

[30] Baryannis, G., Kritikos, K., \& Plexousakis, D. (2017). A specification-based QoS-aware design framework for service-based applications. Service Oriented Computing and Applications, 11(3), 301314

[31] Baryannis, G., \& Plexousakis, D. (2013). WSSL: A Fluent Calculus-Based Language for Web Service Specifications. Paper presented at the 25th International Conference on Advanced Information Systems Engineering (CAiSE 2013), Valencia, Spain.

[32] Baryannis, G., \& Plexousakis, D. (2014). Fluent Calculus-based Semantic Web Service Composition and Verification using WSSL. Paper presented at the 9th International Workshop on Semantic Web Enabled Software Engineering (SWESE2013), co-located with ICSOC 2013, Berlin, Germany. 